\title{
FTMP-based simulations and evaluations of Geometrically-Necessary Boundaries (GNBs) of dislocation
}

\author{
S lhara'*, T Hasebe ${ }^{2}$ \\ 1. Graduate School of Kobe University, Japan \\ 2. Kobe University, Japan
}

\begin{abstract}
The present study targets four representative GNBs (Geometrically Necessary Boundaries) in terms of their unified and quantitative evaluations based on the field theory of multiscale plasticity (FTMP). Discrete dislocation dynamics simulations on GNBs 2, 3, 4 and 7, whose details about the consisting dislocations have been experimentally identified and theoretically evaluated by Hong, Winther, et al., are conducted. Applying the FTMPbased duality diagram representation scheme to the formation processes reveals a systematic interrelationship that further governs the stability of the GNBs, i.e., all the GNBs yield a common tendency to converge ultimately to a single master curve. This leads us to propose a postulate about the stability/instability criterion that involves their dynamic interactions with external disturbances.
\end{abstract}

\section{INTRODUCTION}

Dislocation substructures have been well documented to exhibit diverse configurations in response rather sensitively to the applied loading conditions [1-3]. Since they evolve with or without long-range stress fields and misorientation developments [4], corresponding to their attendant important roles of strain energy storage or release, the morphology-related pieces of information can essentially dominate not only the deformability but also the strength and toughness of the targeted material systems. Therefore, quantification of such dislocation substructures beyond the "density-based" perspectives, including their configurational details and the morphological aspects, is one of the essential issues towards better understanding and, furthermore, rational modeling of crystalline plasticity of metallic materials.

A number of efforts has been dedicated to evaluate dislocation ensembles so far [5-7]. Although these efforts have achieved certain success, no universal approach or scheme that connects them altogether has neither been established nor proposed to date. Dislocation density tensor has recently been frequently used in attempts to capture the evolutionary aspects of many-dislocation systems [8-11]. The tensorial quantity, however, does not inherently contain any information about the configurations, because it is basically determined by the total length of dislocations. This seems to confront the limitation to the conventional crystal plasticity framework.

About 60 years ago, Kazuo Kondo [12-15] proposed the concept of the non-Riemannian plasticity, whose main idea is to capture the defects in materials by torsion and curvature of the crystalline space. In the context of continuum mechanics, the former corresponds to the

${ }^{*}$ Corresponding Author: ihara.shirou@mail.mm4.scitec.kobe-u.ac.jp 
commonly used dislocation density tensor, while the latter to the incompatibility tensor. The dislocation density tensor is equivalent to the density of the geometrically necessary (GN) dislocations $[16,17]$. Since it is classified as the translational type of defects, it vanishes when the net Burgers vectors have canceled each other, as in the case of counting dipole-type dislocation pairs altogether. The incompatibility tensor, on the other hand, can represent spatial dislocation distributions including such multipole-type configurations, since it is defined as the one-more spatial derivative (curl, more precisely) of the dislocation density tensor. We have demonstrated in recent years that the incompatibility tensor can be a powerful tool for evaluating dislocation substructures [20] without any other elaborate procedures, if it is appropriately utilized within the framework provided by the field theory of multiscale plasticity (FTMP) [18-26].

FTMP is a generic theoretical framework that can serve practical solutions to one of the above-mentioned long-standing questions "how can/should we express discrete dislocation ensembles in 3D by continuum languages?" Discrete versions of dislocation dynamics would be a very promising methodology as a "DNS" (direct numerical simulation) technique, more practical and efficient than full atomistic counterparts. The features can be found in [30]. Still, we definitely need the associated continuum descriptions for multiscale perspectives to effectively analyze and evaluate the simulated outputs. Major features, together with complex examples about collapsing wall structures, are found in [20].

Motivated by the theory, the present study attempts to tackle the evaluation of dislocation substructures of complex kinds. Here, we target geometrically necessary boundaries (GNBs), whose detailed structures and the corresponding ideal configurations have been recently identified by Hong, et al. [27] and Winther, et al. [28], respectively. Referring to the basic findings in $[27,28]$, we firstly reproduce the GNBs by using dislocation dynamics code, ParaDiS [29], and examines extensively the simulated dislocation structures based on FTMP. This paper is organized as follows. Section 2 briefly describes the concepts of FTMP-based evaluation. In Section 3, after showing the simulation conditions for the four representative GNBs, we compare the simulated results with the configurations reported in [28]. Section 4 presents the FTMP-based evaluations and discussions on the simulated results in the context of stability/instability criterion, followed by conclusions in Section 5 .

\section{FTMP-BASED EVALUATION}

Mathematically, all the imperfections in a space can be completely expressed by the two differential geometrical quantities in the non-Riemannian geometry, i.e., torsion and curvature [12-15]. They are characterized, respectively, as the closure failure and the rotation of material vectors after parallel displacement encircling the dislocated and/or defected fields, as schematically depicted in Figure 1. The both are higher-ranked tensors, whose contractions ultimately yield second-rank counterparts, known as dislocation density tensor and incompatibility tensor, respectively. The dislocation density tensor is equivalent to the concept of so-called "geometrically-necessary" dislocation density [16, 17]. The more important roles, however, are played by the incompatibility tensor in general, as we have demonstrated in recent years [18-26]. Details about these geometrical quantities are briefly given in the following. 


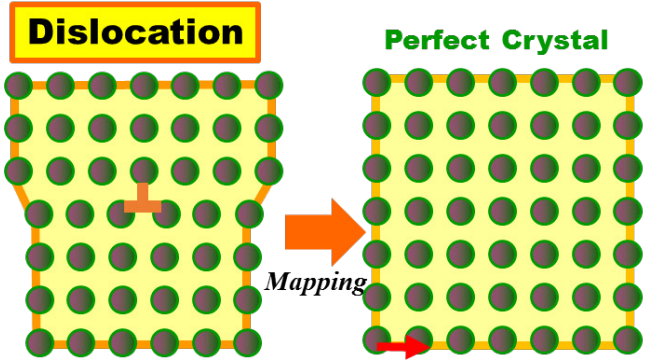

Torsion $S$

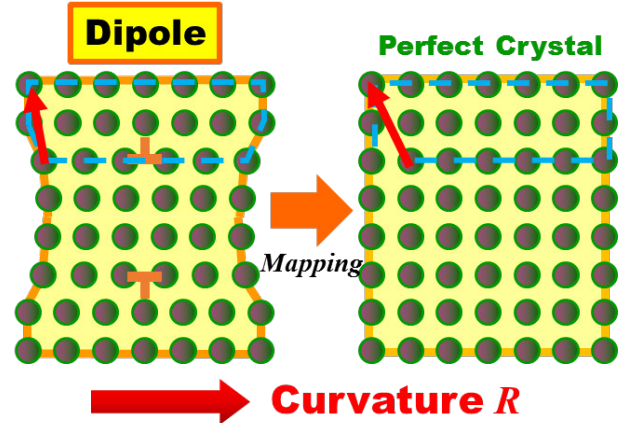

Figure 1: Schematics of torsion tensor and curvature tensor, each characterized as "translational" and "rotational" types of defects, respectively.

\subsection{Incompatibility tensor}

The dislocation density tensor $\alpha_{i j}$ and the incompatibility tensor $\eta_{i j}$ are given by the curl operation of the plastic distortion tensor $\beta_{l j}^{p}$ and the double curl of the plastic strain tensor $\varepsilon_{l n}^{p}$, respectively, i.e.,

$$
\begin{gathered}
\alpha_{i j}=-\epsilon_{i k l} \partial_{k} \beta_{l j}^{p} \\
\eta_{i j}=\epsilon_{i k l} \epsilon_{j m n} \partial_{k} \partial_{m} \varepsilon_{l n}^{p}
\end{gathered}
$$

Since $\varepsilon_{l n}^{p}$ is the symmetrized part of $\beta_{l j}^{p}$, we obtain,

$$
\eta_{i j}=-\epsilon_{i|k l|}\left(\partial_{|k|} \alpha_{j l}\right)
$$

If we consider dislocation system, the surface integral surounding $\alpha_{j l}$ gives the resultant Burgers vectors for the corresponding Burgers circuit as,

$$
b_{l}=\int_{s} \alpha_{j l} \mathrm{~d} S_{j}
$$

where, $\mathrm{d} S_{l}$ is the area in the Burgers circuit.

In this study, we obtain $\alpha_{i j}$ from Equation (3) and $\eta_{i j}$ from Equation (2). Since $\eta_{i j}$ intrinsically contains the pieces of information about how $\alpha_{i j}$ is spatially distributed as in Equation (2), we can obtain also those about the structural aspects of the targeted dislocation systems that cannot be captured by $\alpha_{i j}$ alone. 


\subsection{Flow-evolutionary hypothesis}

In FTMP, the field evolutions in materials is described by the flow-evolutionary hypothesis [19]. This hypothesis is defined as,

$$
\eta_{i j}=\kappa \delta T_{i j}
$$

where $\delta T_{i j}$ is the fluctuation part of the energy-momentum tensor $T_{i j}$, and $\kappa$ is the duality coefficient, which represents a sort of the energy conversion rate. The subscripts $i$ and $j$ denote spatio-temporal component i.e., $i, j=1,2,3$ and 4 (time). Extending the definition of $\eta_{i j}$ to $4 \mathrm{D}$ space-time and assuming spatio-temporal isotropy in Equation (4), we have the temporal component given as [19],

$$
\eta_{K K}=\kappa \delta\left(K+U^{e}\right)
$$

where the index $\mathrm{K}$ denotes the spatial components, i.e., $\mathrm{K}=1,2$ and 3. The right-hand side of Equation (5) consists of the summation of the fluctuation parts of the kinetic energy $K$ and the elastic strain energy $U^{e}$, respectively. When we consider static conditions, Equation (5) reduces to

$$
\eta_{K K}=\kappa \delta U^{e}
$$

This relationship asserts that the excessively stored elastic strain energy is to be converted to the incompatibility-based microscopic degrees of freedom, e.g., those manifested as evolving dislocation substructures. The inverse can include the energy releasing processes, which critically controls the onset of instabilities that eventually can lead to fracture. By plotting both the quantities, $\eta_{K K}$ and $\delta U^{e}$, we obtain duality diagrams, on the other hand, based on which we can further visualize the associated energy flow accompanied by the configurational changes of dislocations taking place within the targeted system.

\section{NUMERICAL EXPERIMENTS}

\subsection{Simulation models}

Figure 2 shows the initial and ideal configurations of GNBs reported by Winther, et al. [28], together with their experimental observations. Here, we classify the GNBs in terms of the consisting Burgers vectors according to [28], i.e., the GNB2 is composed of 2 sets of Burgers vectors and it forms hexagonal network after the reaction of the two, while the GNBs 3 and 7 have 3 sets of Burgers vectors and their ideal configurations are the ladder-like patterns. The GNB4, on the other hand, has a combined morphology of the GNBs 3 and 7, and its ideal configuration is also obtained by the corresponding combination.

Figure 3 indicates the GNB models, where the definition of the dislocation components follows [28]. Here, the targeted material is assumed to be Al (FCC), corresponding to the experiment in [27], with the shear modulus $25.5 \mathrm{GPa}$, Poisson's ratio 0.34, and the magnitude of Burgers vector $2.86 \times 10^{-10} \mathrm{~m}$. The wall models for the four representative GNBs, i.e., 2, 3, 4 and 7, are displayed in Figure 3, where periodic boundary conditions for all the directions are assumed, combined with the Fast Multipole Method (FMM) [29] incorporated in the ParaDiS

code 
(ver.2.5.1). Note, since all the edges of the walls are isolated in the simulation cells because of the modeling restrictions in utilizing the FMM, we set the evaluation range as depicted in Figure 3 (c) for obtaining necessary quantities, e.g., the stress and the incompatibility tensor fields.

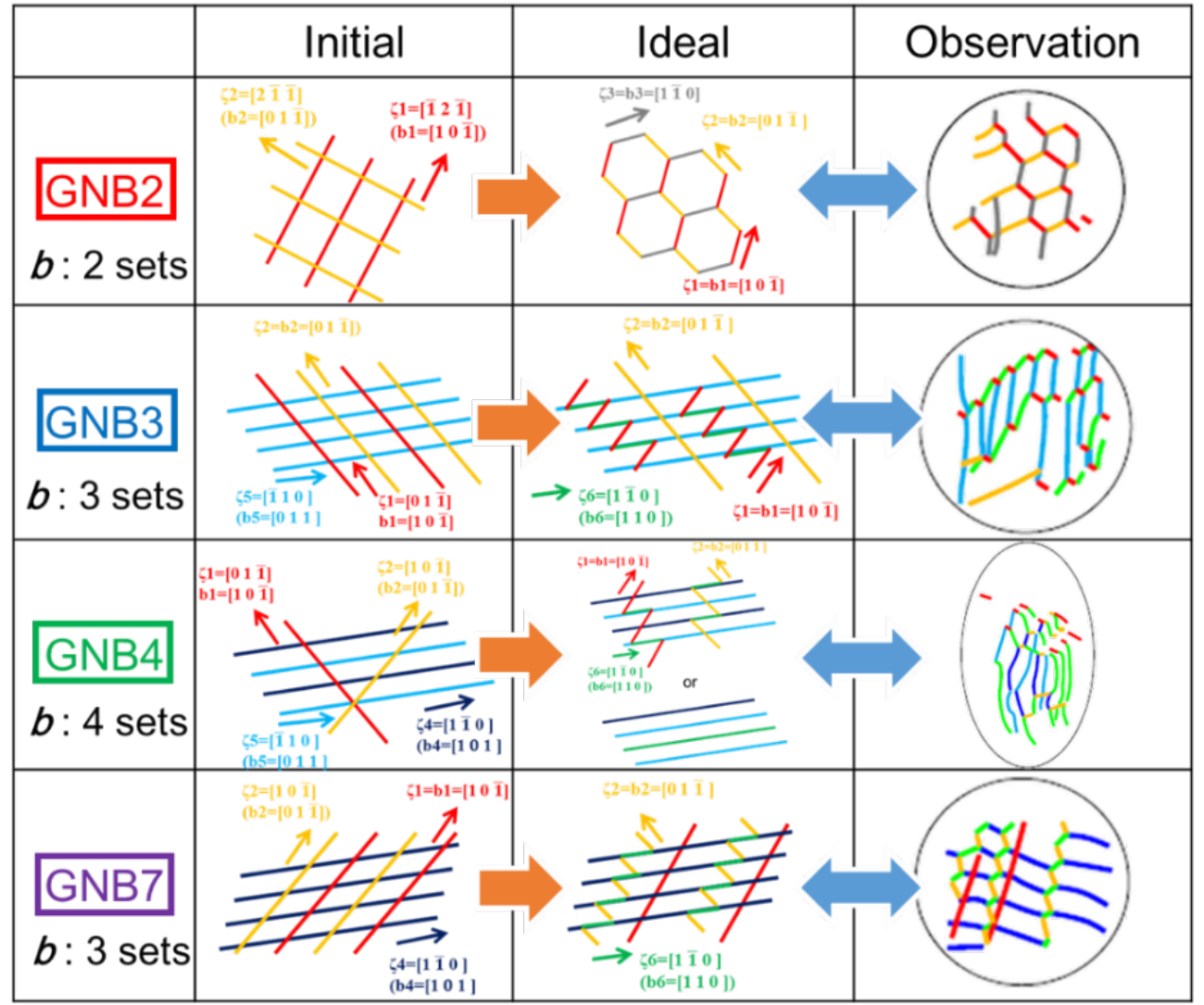

Figure 2: Schematic illustration of initial and final dislocation configurations for four representative GNBs, consisting of mutually different numbers and combinations of Burgers vectors, together with experimentally observed counterparts by Winther, et al [28].

The dislocation densities for the initial configurations are $7.05 \times 10^{14} \mathrm{~m}^{-2}$ (GNB2), $7.45 \times 10^{14} \mathrm{~m}^{-2}$ (GNB3 and GNB7), and $8.89 \times 10^{14} \mathrm{~m}^{-2}$ (GNB4), respectively. Here, we apply no external stress such that the GNBs organize spontaneously into their ideal configurations of their own.

\subsection{GNB-formation}

Figure 4 compares the initial and final configurations for the four GNBs obtained in the present series of simulations, together with the corresponding ideal and experimentallyobserved configurations by Winther et al [28]. The comparison demonstrates that the simulated final configurations agree well with the ideal counterparts. The reactions taken place during the configurational changes are roughly the same as that have been supposed by Winther et al [28]. The GNB2 forms hexagonal networks as a result of the formation of 


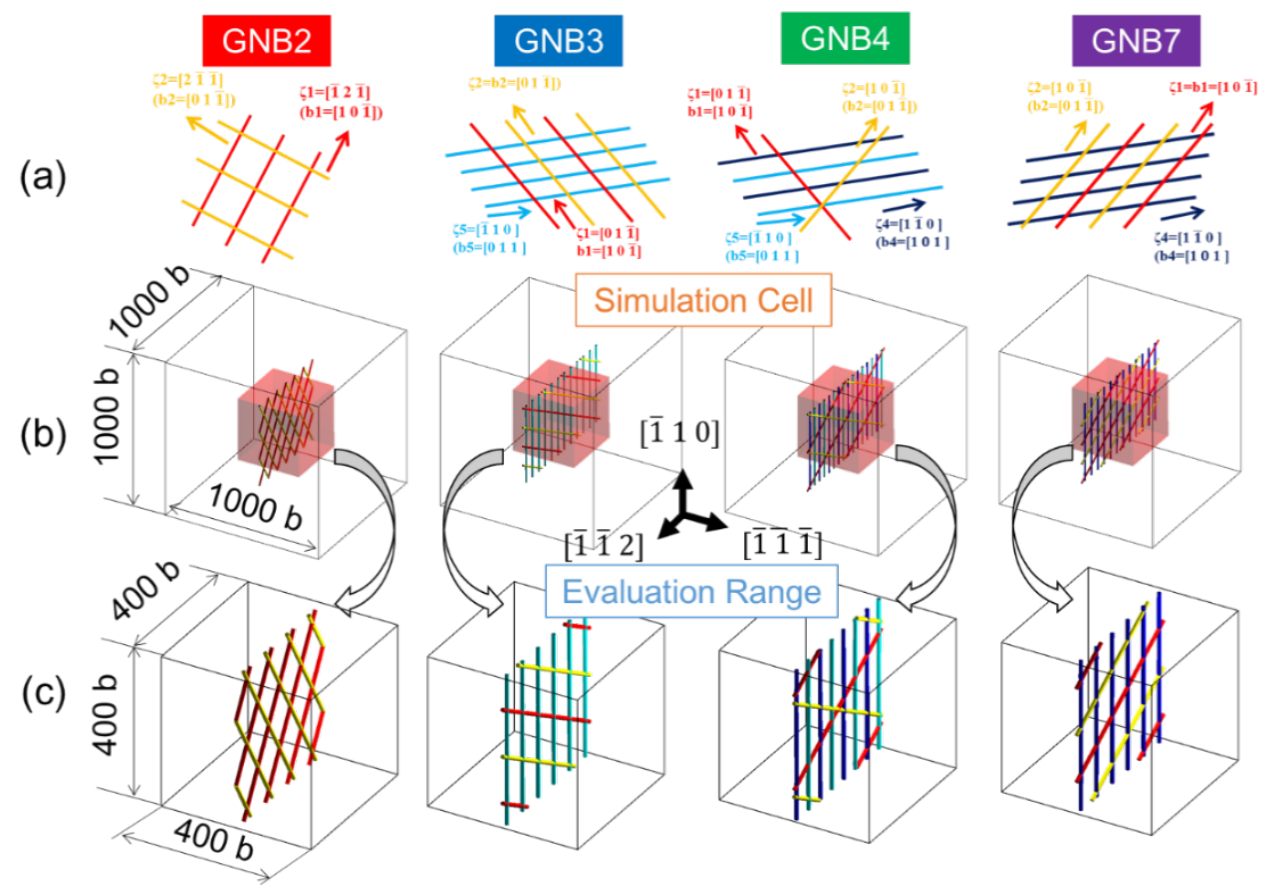

Figure 3: Simulation models of four GNBs, showing initial configurations with periodic boundary conditions, together with evaluation regions for stress and incompatibility calculations.
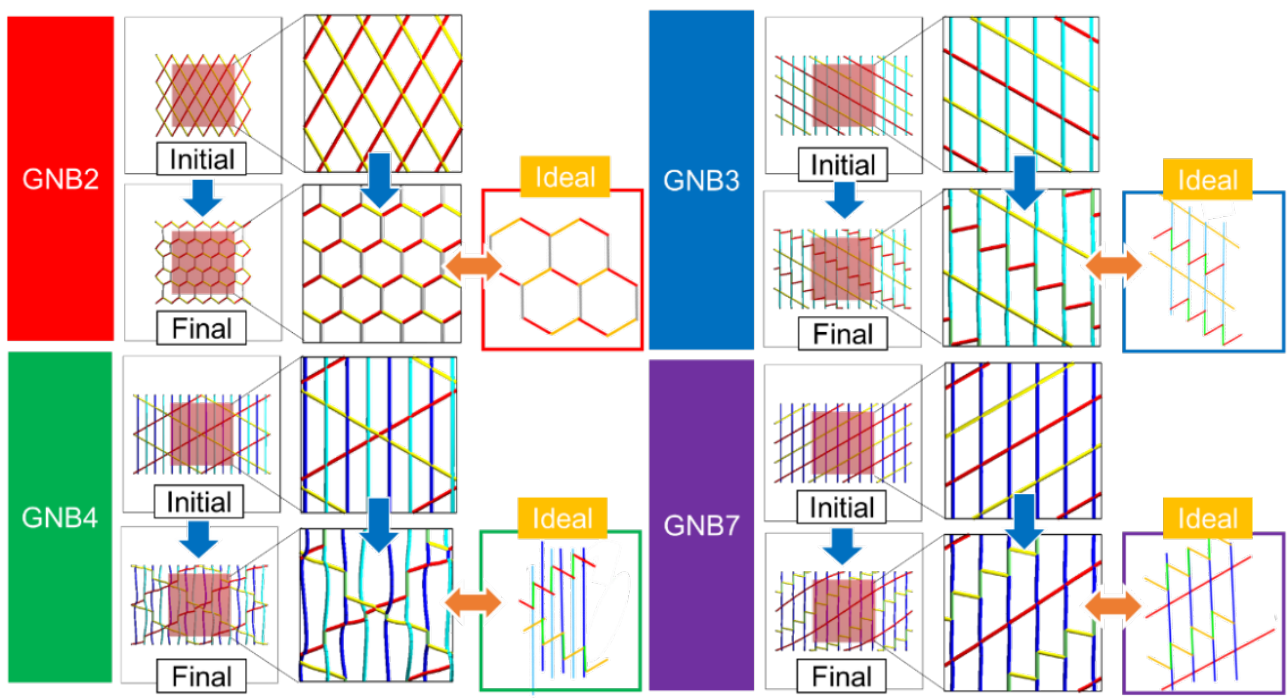

Figure 4: Simulated initial and final configurations for GNBs 2, 3, 4 and 7, compared with those predicted by Winther, et al [28] 
junctions (colored in grey in Figure 4) between two Burgers vectors indicated in Figure 3(a), while the GNBs 3 and 7 ultimately yield ladder-like patterns with junctions (green) produced by the interactions of two Burgers vectors among three. The GNB4, consisting of two sets of junctions (both in green) out of the four Burgers vectors, on the other hand, exhibits relatively complex morphology, not a simple superposition of those for the GNBs 3 and 7, as expected in Section 3.1, i.e., it all consists of curved segments, brought about by its own highest density of dislocations. The stress fields produced by the high density thus slightly differentiate the final morphology from the ideal one.

\section{RESULTS AND DISCUSSIONS}

\subsection{GNB-formation}

Figures 5 and 6 indicate variations of the elastic strain energy fluctuation $\delta U^{e}$ and the incompatibility $\eta_{K K}$ with elapsed time for the four GNBs simulated, respectively. Comparing the two sets of variations, we confirm mutually similar overall trends, except for the GNB4, i.e., initial sharp drops in the GNB2, while almost constant variations for the GNBs 3 and 7. The GNB4, on the other hand, shows conspicuous oscillation in the strain energy fluctuation following an initial sharp rise, while it yields relatively moderate ups and downs in the incompatibility change. The oscillating strain energy fluctuation stems from its complex configurational changes, mainly due to the junction formations taking place at the intersections, as can be confirmed in Figure 3, resulting in the formations of a number of crooked segments that cause highly fluctuating stress field. The attendant configurational changes, on the other hand, do not significantly affect the incompatibility variation.

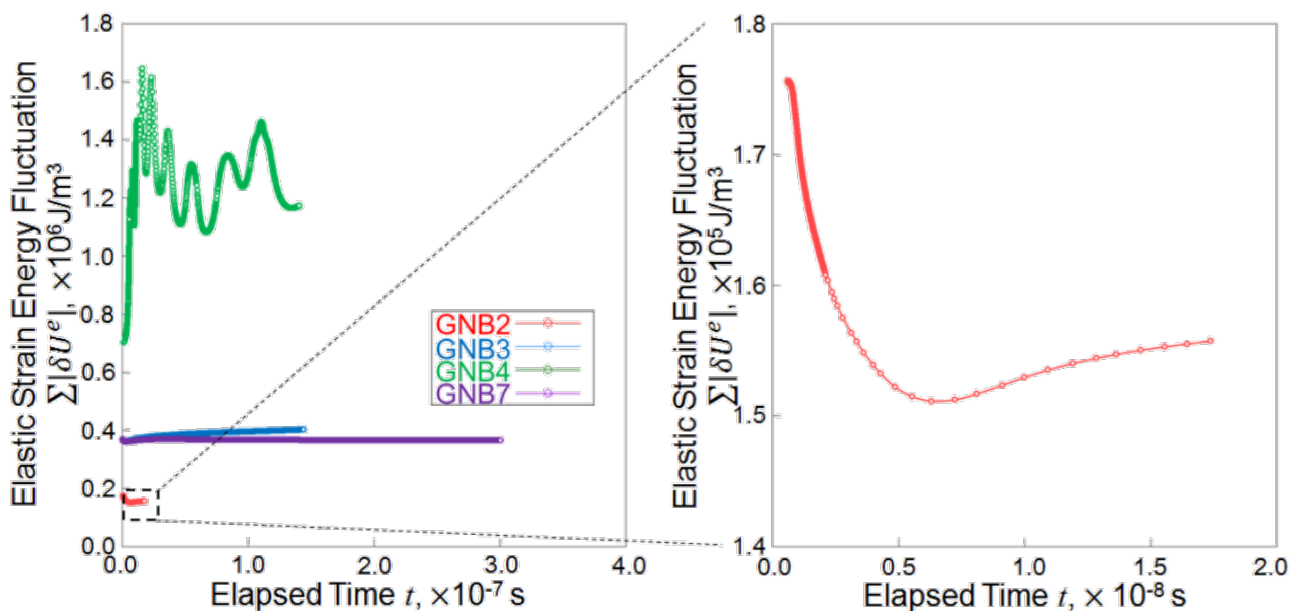

Figure 5: Variation of elastic strain energy fluctuation with elapsed time, comparing four GNBs simulated

By correlating the above two quantities, $\delta U^{e}$ and $\eta_{K K}$, we obtain the corresponding duality diagrams for all the GNBs simulated, as displayed in Figure 7. Here, the large solid circles indicate the respective final steps. As demonstrated in the diagram, it can further clarify the mutually distinctive trends among the four GNBs, corresponding to Figures 5 and 6 
described above. The GNB2, with a regular hexagonal network, is located in the far lowerleft of the diagram after a sharp drop in $\eta_{K K}$, demonstrating it being the lowest energy configuration among others, whereas the relatively irregular GNB4 is ultimately situated in the far upper-right, following sharply drifting $\delta U^{e}$ brought about by comparably large stress field fluctuations. The GNBs 3 and 7, on the other hand, are located between the two, after showing moderate variations.

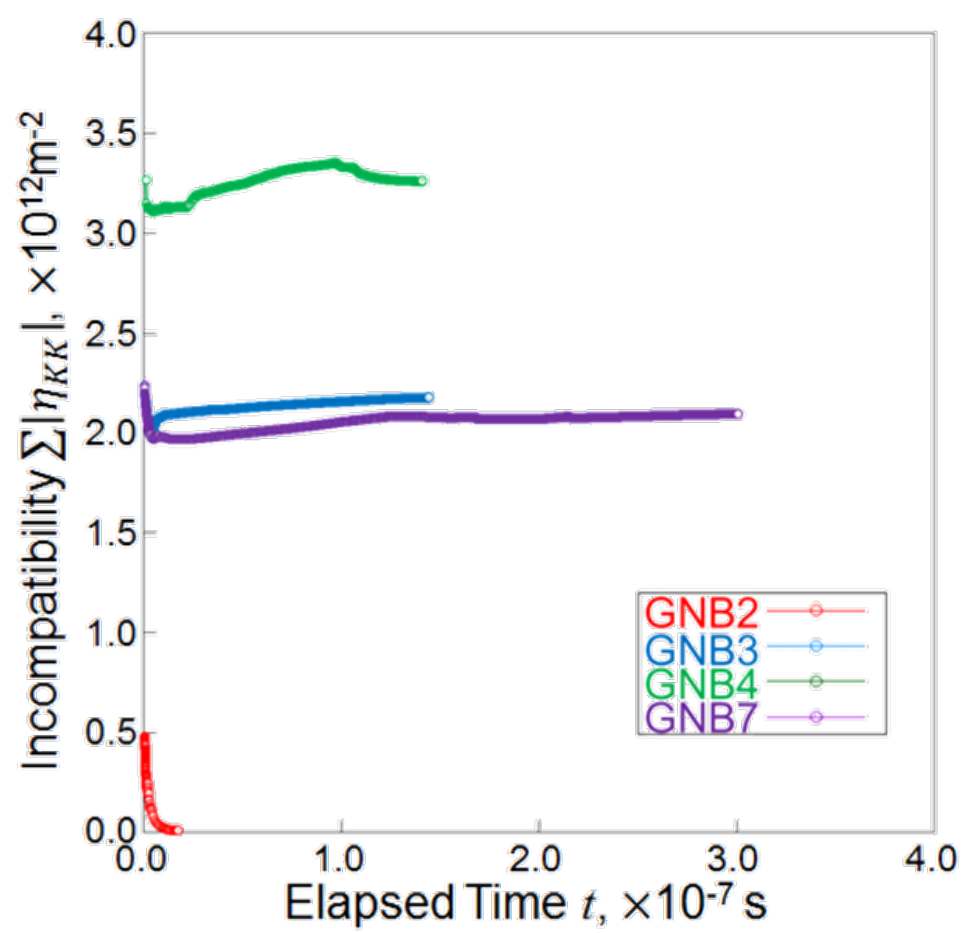

Figure 6: Variation of incompatibility with elapsed time, comparing four GNBs simulated.

Another marked implication derived from the diagrams is a systematic interrelationship found among the four GNBs, i.e., all the final configurations clearly exhibit a tendency to be aligned on a single master curve, indicating a system of the most stable configurations. To further examine this, we next discuss the effect of the kinetic energy contributions to the duality diagram representations, regarding it as a sort of external perturbations.

We define the kinetic energy for a dislocation segment as,

$$
K=\frac{1}{2} \rho b^{3} l v^{2}
$$

where $\rho$ is the density, $b$ is the magnitude of Burgers vector, $l$ is the dislocation segment length (unit of $b$ ), and $v$ is the speed of dislocation node. Here, the mass of dislocations is assumed to be equal to that of the removed atoms by the dislocations [31, 32]. 


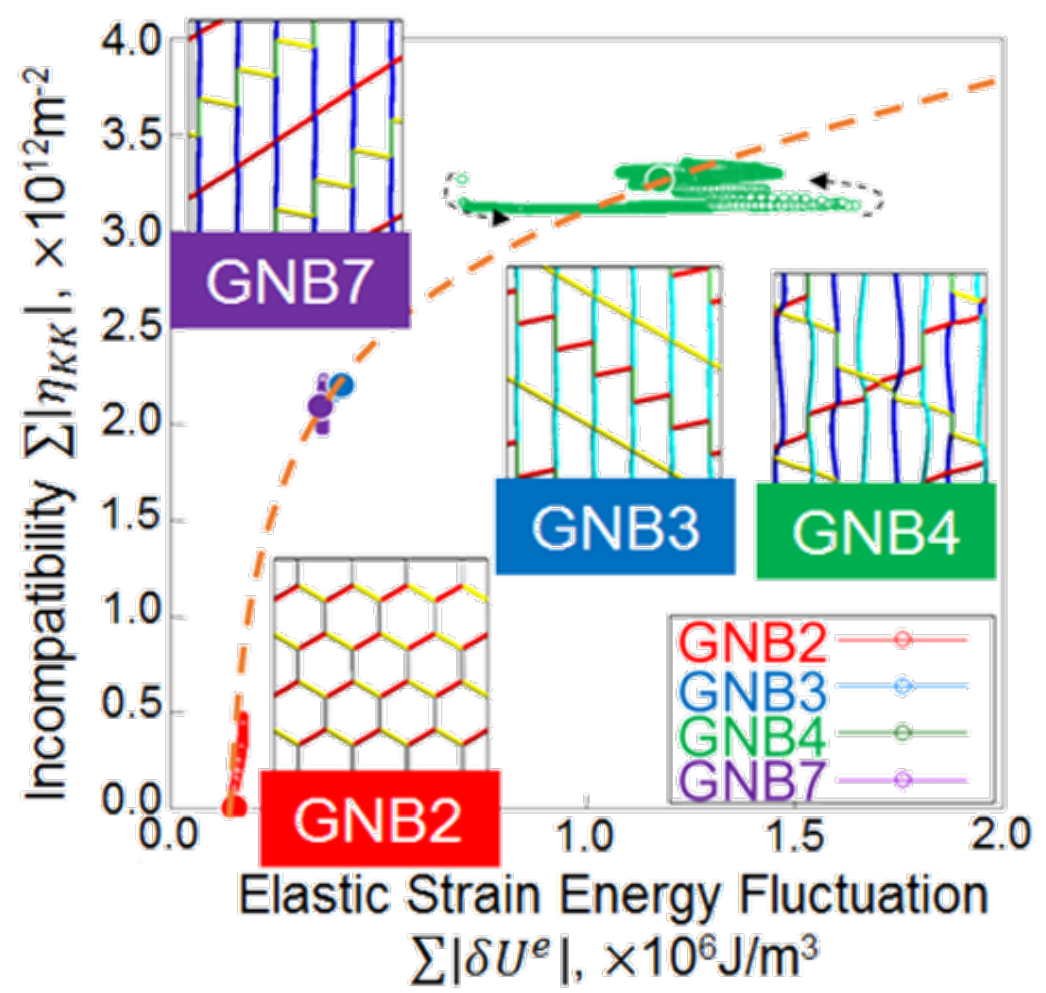

Figure 7: Duality diagrams for four GNBs simulated, where final states are indicated by large sold circles, respectively.

In the present series of simulations, the kinetic energy is negligibly small compared with $\delta U^{e}$. Therefore, we here virtually enhance their contributions by multiplying a coefficient $\alpha=$ $1 \times 10^{7}$ to examine their potential effects on the duality diagram representations.

Figure 8 displays the duality diagrams taking account of thus enhanced kinetic energy contributions to the abscissa. As readily found in the modified diagrams, in comparison with Figure 7, the contributions of the kinetic energy greatly vary depending on the GNB types, i.e., the GNB2 exhibits exclusively large variation, the GNBs 3 and 7 show the smallest, whereas the GNB4 yields conspicuous drift similar to the correlation with $\delta U^{e}$ only.

Since the multiplying factor is set in common to all the GNBs, this inverse trend to $\delta U^{e}$ observed in Figure 7 logically implies the following. The GNB2 and GNB4 have experienced larger additional perturbations than the GNBs 3 and 7 . Again referring to the duality diagrams in Figure 7 afresh, we notice an important overall trend, i.e., the GNB2 tends to cope with the external disturbance by large configurational changes, manifested as the largest variation in the incompatibility, whereas the GNB4 tries to withstand rather firmly, with the minimum incompatibility changes.

From the above discussion, we derive a postulate as follows. All the GNBs ultimately tends to converge to a single series of configurations (those aligned on a master curve) after relaxation from disturbed states, but with distinct ways depending on the locations on the 
diagram. The feature can be a controlling mechanism for their gaining stabilities that is hidden behind the organic interrelationship among distinct GNBs in the energy-configuration contexts. This further motivate us to propose tentatively a new stability/instability criterion for the GNBs based on it.

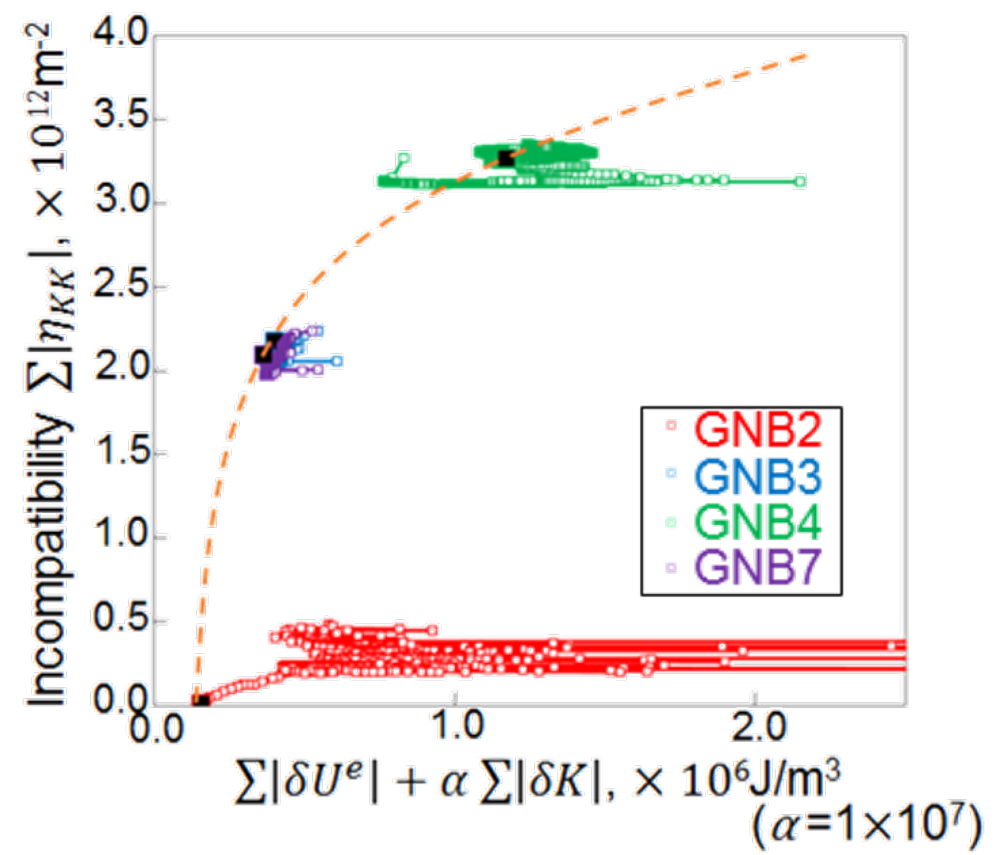

Figure 8: Same results as Fig.7 but with virtually-pronounced contributions of kinetic energy overplotted on final states (solid black squares).

Figure 9 illustrates the schematics of the new stability/instability criterion, by representing an evaluation procedure based on the duality diagrams in Figure 7 as an example case.

If the GNB2 is disturbed by $\delta\left(U^{e}+K\right)$, e.g., via collisions with in-coming dislocation fluxes, (1) in Figure 9), the perturbed wall will eventually relax and the extra energy will then be converted into that for the configurational changes. This relaxed state on the duality diagram will be placed back on the master curve (2) if the wall structure is stable enough. When the same event occurs against the GNB4 (3), on the other hand, its relaxed structure will also come back to the master curve, but in this case, accompanied by relatively small incompatibility change (4). By comparing the incompatibility changes (5), together with how fast the perturbed states regain the master state, we can judge the "degree" or the "quality" of the stability of the targeted wall structures. 


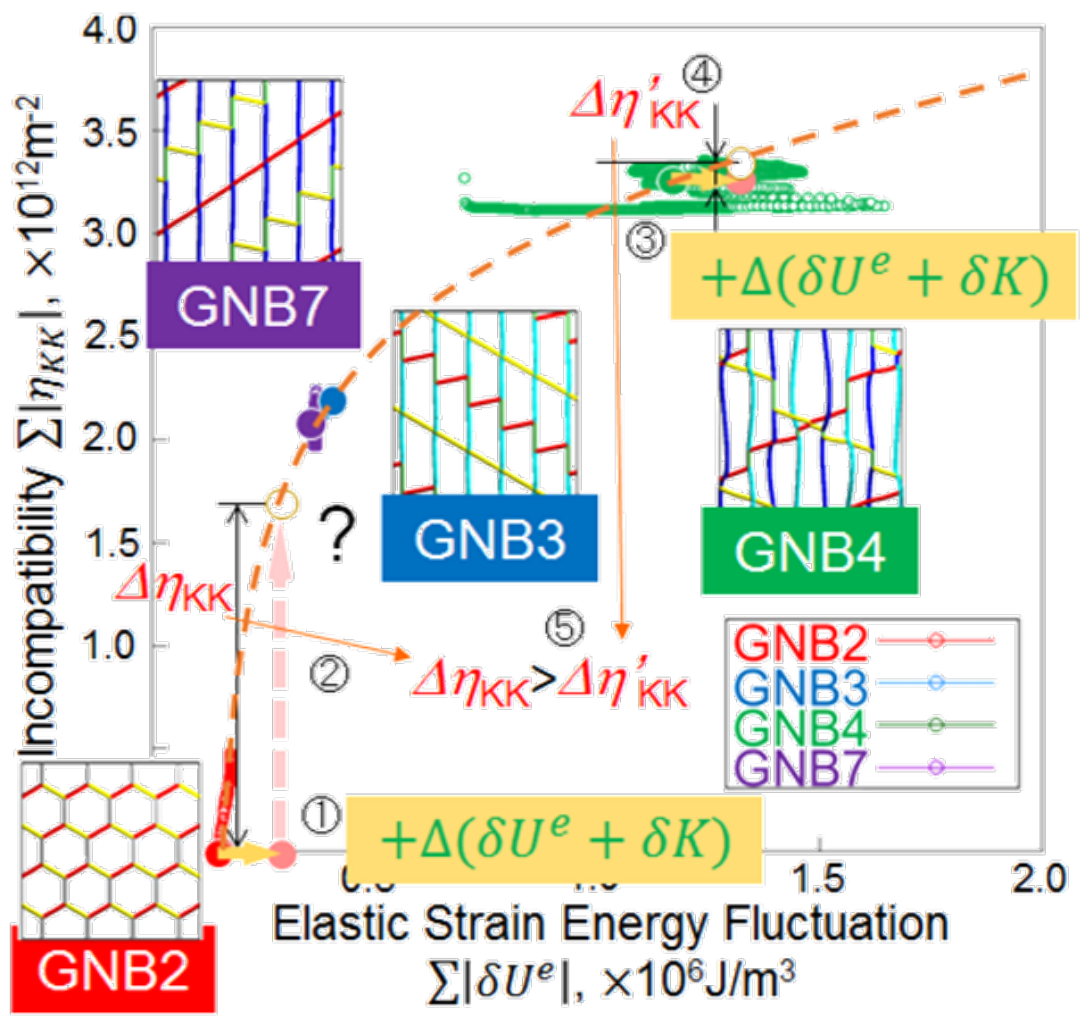

Figure 9: Schematics of new stability/instability criterion, showing evaluation procedures by comparing anticipated incompatibility responses against a prescribed external energy disturbance on duality diagrams.

\subsection{Verification}

To partially verify the above postulate, we collide a dislocation loop against the GNB2 as an external disturbance. Figure 10 shows the simulation setup, where a Frank-Read (F-R) source is situated away from the wall. We apply the external stress $\sigma_{23}=1.0 \mathrm{GPa}$ so that the source generates a dislocation loop. The evaluation range and the other conditions are the same as those in Section 3.1.

Figure 11 shows the obtained results, demonstrating ultimately a topological change in the central portion of the GNB2. Looking into details of the interaction via the three snapshots, we observe that the right vertical segment of the central hexagonal network (colored in grey) is subjected to (a) a cross slip as a result of the interaction. This further brings about (b) another loop generation from the wall, eventually exhibiting (c) local annihilation in the lower hexagonal unit. The incident dislocation loop, on the other hand, keeps expanding, causing no more reaction with GNB2.

Overplotting the corresponding duality diagram to the above series of processes on Figure 7, we obtain Figure 12(a). The open orange circle indicates the tentative final state with disregarding the presence of the incident dislocation, showing apparently the diagram is going out of the master curve. To confine ourselves to evaluate the net configurational change of the wall structure itself, we remove the segments protruded from the wall-deemed region. 
Figure 12(b) shows the magnified duality diagram after the removal operation, demonstrating that the plot for the final state is shifted leftward and is eventually placed rather exactly on the master curve, as depicted by a white arrow in the inset.

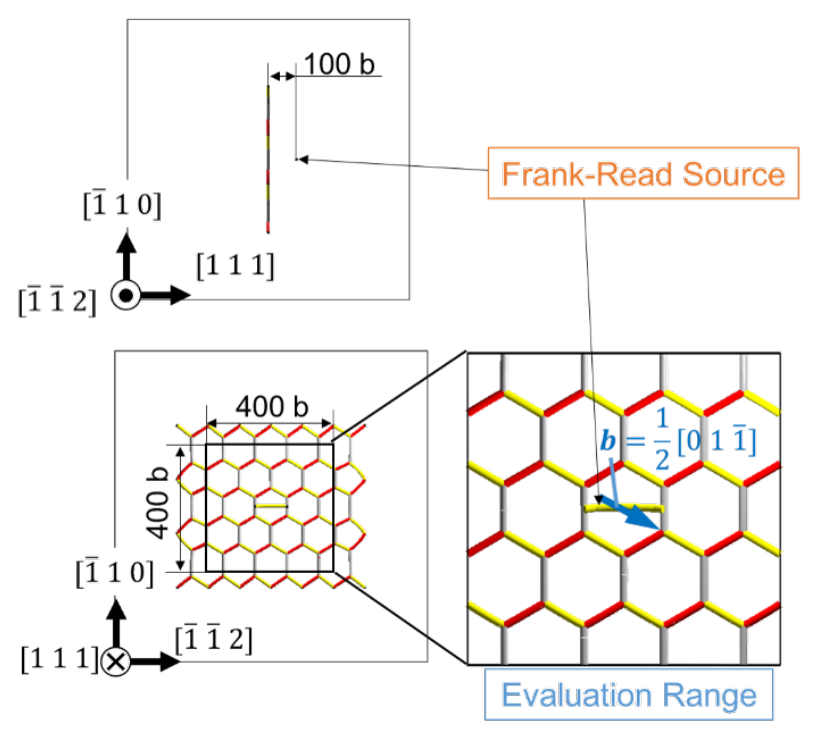

Figure 10: Simulation set up for verifying stability/instability evaluation scheme, where a dislocation expanded from a Frank-Read source is to be collided against GNB2 model as external perturbation

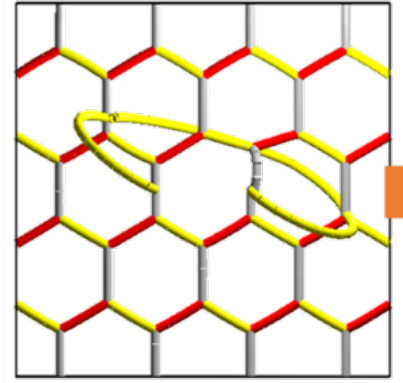

(a)

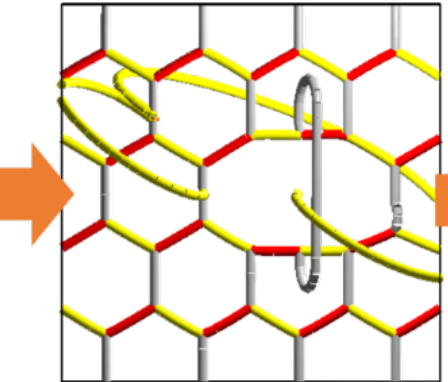

(b)

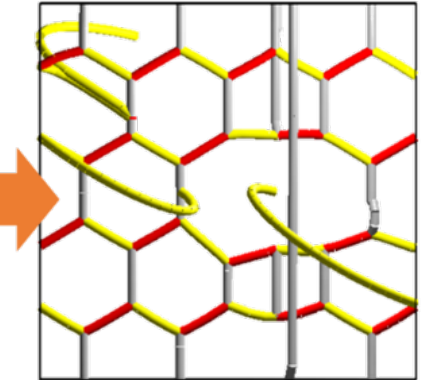

(c)

Figure 11: Snapshots for details of interaction with incoming dislocation, giving rise to topological changes of network configuration in GNB2.

The resultant morphology is further compared with the experimentally-observed one for GNB2 in Figure 13, together with that for the GNB4 [27]. We can confirm some similar configurations between the two (1) in Figure 13). Since the experimental GNB2 frequently contains such greatly disturbed configurations, we conjecture that it has been experienced 
frequent external disturbances and coped with them by large configurational changes. The GNB4, on the other hand, exhibits relatively "solid" network configuration (2), serving an indirect evidence of the "stiff" structure against external perturbations.

The duality diagram representation demonstrated in Figure 12, together with the leveraging comparison with the experiments presented above, we tentatively conclude that the current new postulate on the stability/instability evaluation criterion is partially verified.

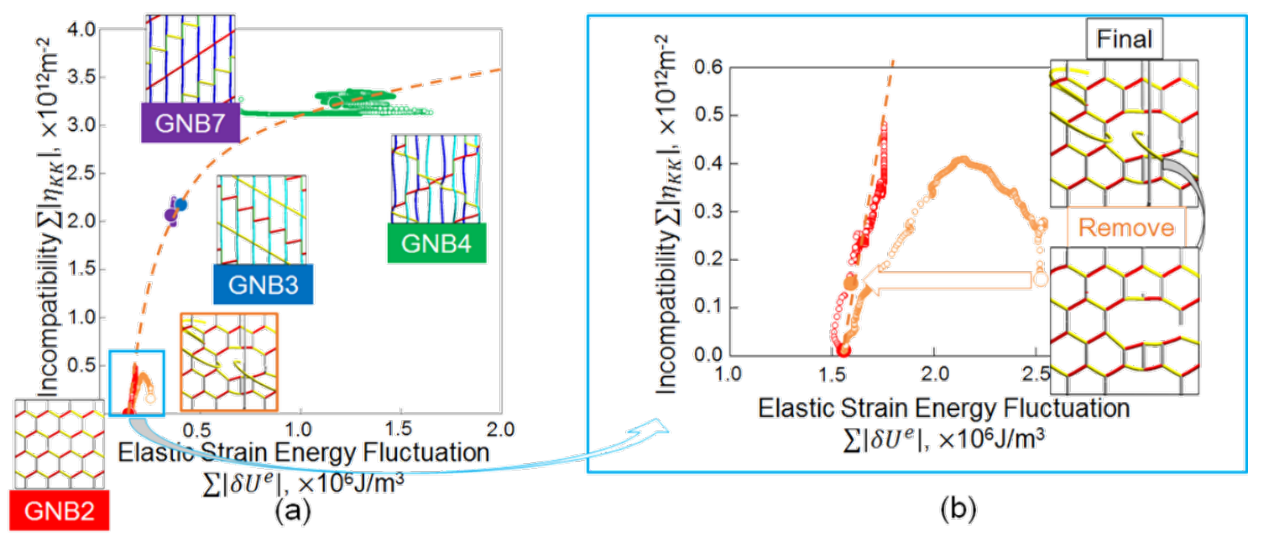

Figure 12: Duality diagram representation for perturbed GNB2 (a) overplotted on Figure 7, and (b) magnified view indicating before and after removal operations for out-of-wall segments in strain energy calculation for final configuration.

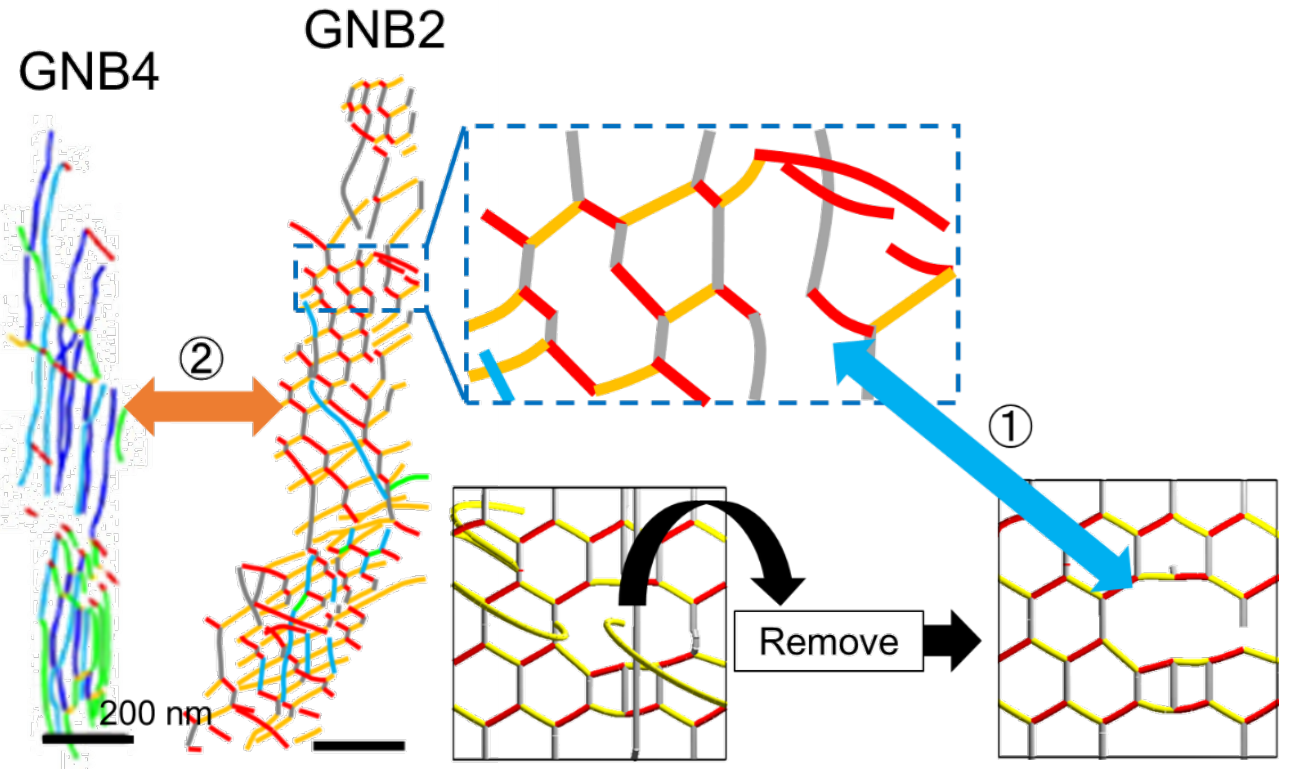

Figure 13: Comparison of disturbed configurations for GNB2 between simulation and experiment, together with GNB4 in experiment [27]. 


\section{CONCLUSIONS}

Rational as well as practically-feasible treatments of spatio-temporally distributed discrete dislocation ensembles have been a long-standing issue and still a challenging problem inevitable in completion of multiscale modeling of metallic materials. Unlike 2D cases, which can be simply reduced down to continuously-distributed density function-like pictures, 3D counterparts need to deal with its configurational complexities more or less explicitly. Among others, quantitative stability/instability assessments of wall structures are critically important, in the sense that they substantially dominates both the micro/macro mechanical properties of the material systems concerned.

The present study tackled the above based on FTMP (Field Theory of Multiscale Plasticity), focusing on their continuum descriptions in the flow-evolutionary context, where the duality diagram representation scheme is mainly used. The scheme enables visualizations of the energy flow associated with the configurational changes of the targeted manydislocation systems.

Here, the four GNBs are successfully reproduced from the corresponding initial configurations based on a series of dislocation dynamics simulations by using the Para-Dis code. The simulated results demonstrate that all the duality diagrams tend to converge on a single smooth curve, strongly implying an organic interrelationship should exist among at least the present four GNBs in the flow-evolutionary contexts. The study also examines virtual contributions of the kinetic energy fluctuation to the corresponding incompatibility evolutions via the duality diagram scheme and some dynamic interactions with an in-coming dislocation for the GNB2 wall model. The former motivates us to postulate a new stability/instability criterion against the dislocation wall structures, which is expected not to be limited to the GNBs dealt with in the present study, while the latter partially verifies the proposed new hypothesis, although it deserves further examinations.

\section{REFERENCES}

[1] Suresh, S., Fatigue of Materials, 1998: Cambridge University Press.

[2] Tanaka, E., Murakami, S. and Ooka, M., Dependence of train-Hardening under Multiaxial Non-Proportional Cyclic Plastic Deformation on Geometry History of Plastic Strain Trajectory, Trans. JSME A, 1985. 51(468): p. 1941-1950.

[3] Kuhlmann-Wilsdorf, D. and Hansen, N., Geometrically Necessary, Incidental and Subgrain Boundaries, Scripta Metall. Mater. 1991. 25(7): p. 1557-1562.

[4] Mughrabi, H., Ungar, T., Kienle, W. and Wilkens, M., Long-Range Internal Stress and Asymmetric X-Ray Line Broadening in Tensile Deformed [001]-Oriented Copper Single Crystals, Phil. Mag. A, 1986. 53(6): p. 793-813.

[5] Zaiser, M., Bay, K. and Hahner, P., Fractal Analysis of Deformation-Induced Dislocation Patterns, Acta. Mater., 1999. 47(8): p. 2463-2476.

[6] Hussein, A. M. and El-Awady, J. A., Quantifying Dislocation Microstructure Evolution and Cyclic Hardening in Fatigued Face-Centered Cubic Single Crystals, J. Mech. Phys. Solids, 2016. 91: p. 126-144.

[7] Steinberger, D., Gatti, R. and Sandfeld, S., A Universal Approach Towards Computational Characterization of Dislocation Microstructure, JOM, 2016. 68(8): p. 2065-2072. 
[8] Sandfeld, S. and Po, G., Microstructural Comparison of the Kinematics of Discrete and Continuum Dislocation Models, Modelling Simul. Mater. Sci. Eng., 2015. 23: 085003(27pp).

[9] Jones, R. E., Zimmerman, J. A., Po, G. and Mandadapu, K. K., Comparison of Dislocation Density Tensor Fields Derive from Discrete Dislocation Dynamics and Crystal Plasticity Simulations of Torsion, J. Mater. Sci. Res., 2016

[10] Ortiz, M., and Repetto, E. A., Nonconvex Energy Minimization and Dislocation Structures in Ductile Single Crystals, J. Mech. Phys. Solids, 1999. 47: p. 397-462.

[11] Edmiston, J., Steigmann, D.J., Johnson, G.J. and Barton, N., A Model for ElasticViscoplastic Deformations of Crystalline Solids Based on Material Symmetry: Theory and Plane-Strain Simulations, Int. J. Eng. Sci., 2013. 63: p. 10-22.

[12] Kondo, K., Non-Riemannian Geometry of Imperfect Crystals from a Macroscopic Viewpoint, RRAG Memoirs, 1955. 1(D-I): p. 458-469. (RAAG Memoirs of Unifying Study of Basic Problems in Engineering and Physical Science by Means of Geometry (ed: Kondo. K.), Gakujutsu Bunken Fukyu-kai, Tokyo).

[13] Kondo, K., Energy at Plastic Deformation and the Criterion for Yielding, RAAG Memoirs, 1955. 1(D-III): p. 484-494.

[14] Kondo, K., Derivation of the Differential Equations of Yielding from General Variational Criteria in Analogy with General Relativity Theory and an Extension Thereof to Problems involving Critical Temperature, RAAG Memoirs, 1962. 3(D-XII): p. 215-227.

[15] Amari, S., A Theory of Deformations and Stresses of Ferromagnetic Substances by Finsler Geometry, RAAG Memoirs, 1962. 3: p. 257-278.

[16] Ashby, M.F., Deformation of Plastically Non-Homogeneous Materials, Phil. Mag., 1970. 21: p. 399-424.

[17] Fleck, N.A., and Hutchinson, J.W., A Reformulation of Strain Gradient Plasticity, J. Mech. Phys. Solids, 2001. 49: p. 2245-2271.

[18] Hasebe, T., Continuum Description of Inhomogeniousely Deforming Polycrystalline Aggregate based on Field Theory, IUTAM Symposium on Mesoscopic Dynamics of Fracture Process and Materials Strength. Eds. H. Kitagawa and Y. Shibutani, Kluwer Academic Publishers, 2004: p. 381-390.

[19] Hasebe, T., Sugiyma, M., Adachi, H., Fukutani, S. and Iida, M., Modeling and Simulations of Experimentally-Observed Dislocation Substructures Based on Field Theory of Multiscale Plasticity (FTMP) Combined with TEM and EBSD-Wilkinson Method for FCC and BCC Poly/Single Crystals, Mater. Trans., 2014. 55(5): p. 779-787.

[20] Hasebe, T. and Naito, T., FTMP-based 4D Evaluations of Discrete Dislocation Systems, New Frontiers of Nanometals, Eds. S. Faester, et al. (Proc. 35th Ris $\varnothing$ int. Symp. on Maters. Sci.), 2014. p. 305-312.

[21] Yamada, M., Hasebe, T., Chen, J.-S. and Guan, P.-C., Reproducing Kernel Based Evaluation of Incompatibility Tensor in Field Theory of Plasticity, IMMIJ (Interaction and Multiscale Mechanics: An Int. J.), 2008. 1(4): p. 423-435. 
[22] Aoyagi, Y. and Hasebe, T., New Physical Interpretation of Incompatibility Tensor and Its Application to Dislocation Substructure Evolution, Key Materials Engineering, 2007. 340(341): p. 217-222.

[23] Yamada, M., Hasebe, T., Tomita, Y., Onizawa, T., Reproducing Kernel Based Evaluation of Incompatibility Tensor in Field Theory of Plasticity, IMMIJ (Interaction and Multiscale Mechanics: An Int. J.), 2008. 1(4): p.437-448.

[24] Hasebe, T., Multiscale Crystal Plasticity Modeling based on Field Theory, CMES, 2006. 11(3): p. 145-155.

[25] Hasebe, T., Interaction Fields Based on Incompatibility Tensor in Field Theory of Plasticity-Part I: Theory-, IMMIJ (Interaction and Multiscale Mechanics: An Int. J.), 2009. 2(1): p. 1-14.

[26] Hasebe, T., Interaction Fields Based on Incompatibility Tensor in Field Theory of Plasticity -Part II: Application-, IMMIJ (Interaction and Multiscale Mechanics: An Int. J.), 2009. 2(1): p. 15-30.

[27] Hong, C., Huang, X. and Winther, G., Dislocation Content of Geometrically Necessary Boundaries Aligned with Slip Planes in Rolled Aluminium, Phil. Mag. A, 2013. 93(23): p. 3118-3141.

[28] Winther, G., Hong, C. and Huang, X., Low-Energy Dislocation Structure (LEDS) Character of Dislocation Boundaries Aligned with Slip Planes in Rolled Aluminium, Phil. Mag., 2015. 95(13): p. 1471-1489.:

[29] Arsenlis, A., Cai, W., Tang, M., Rhee, M., Oppelstrup, T., Hommes, G., Pierce, T. G. and Bulatov, V. V., Enabling Strain Hardening Simulations with Dislocation Dynamics, Modelling Simul. Mater. Sci. Eng., 2007. 15(6): p. 553-595.

[30] Bulatov, V.V., Cai, W., Computer Simulations of Dislocations, 2006: Oxford University Press.,

[31] The Japan Institute of Metals and Materials, Theory of Dislocations: Its Applications to Metallurgy (in Japanese), 1971: Maruzen.

[32] Meyers, M. A., Dynamic Behavior of Materials, 1994: Wiley. 